\title{
Use of the D Test Method to Detect Inducible Clindamycin Resistance in Coagulase Negative Staphylococci (CoNS)
}

\author{
Leandro Reus Rodrigues Perez ${ }^{1}$, Juliana Caierão ${ }^{1}$, Ana Lúcia Souza Antunes ${ }^{1,2}$ and Pedro Alves d'Azevedo ${ }^{1}$ \\ ${ }^{1}$ Foundation of Federal Clinical Medical School of Porto Alegre; ${ }^{2}$ Pharmacy School, \\ Federal University of Rio Grande do Sul, Porto Alegre, RS, Brazil
}

\begin{abstract}
According to the National Committee for Clinical Laboratory Standards (NCCLS, 2004), a method to evaluate the inducible clindamycin resistance in accordance with an approach of the disks of erythromycin and clindamycin the $D$ test - has been reported. We analyzed the performance of this method in 200 coagulase negative staphylococci (CoNS) strains obtained from blood cultures of hospitalized patients at a general hospital in Southern Brazil. Twenty-seven clinical isolates with suitable profile (erythromycin-resistant and clindamycin-susceptible) were evaluated for the $D$ test realization. Thus, only 5 CoNS were $D$ test positive. The $D$ test method showed to be simple and an important technique in the detection of inducible clindamycin resistance.
\end{abstract}

Key-Words: Clindamycin, resistance, $\mathrm{D}$ test.

The determination of antimicrobial susceptibility of a clinical isolate is often crucial for the optimal antimicrobial therapy of infected patients. This is particularly important considering the increase of resistance and the emergence of multidrug-resistant microorganisms [1-3]. Several authors have screened clinical isolates of erythromycin-resistant Staphylococcus aureus and coagulase negative staphylococci (CoNS) for genes encoding resistance to macrolides, lincosamides and streptogramins type $\mathrm{B}\left(\mathrm{MLS}_{\mathrm{B}}\right)$ [4-10].

Resistance to macrolides (e.g. erythromycin) can occur by two different mechanisms: efflux due to macrolide streptogramin resistance ( $m s r A$ gene) and ribosome alteration due to erythromycin ribosome methylase (erm gene) [11,12]. Macrolide resistance due to efflux encoded by $m s r A$ has been more prevalent in CoNS than in S. aureus [13].

Different mechanisms of acquired MLS resistance have been found in Gram-positive bacteria [11,12]. The first mechanism of resistance to macrolide described was due to posttranscriptional modifications of the 23S rRNA by the adenine- $N$-6-methyltransferase. Target modification alters a site in 23S rRNA common to the binding of MLS $_{\mathrm{B}}$ antibiotics. Modification of the ribosomal target confers cross-resistance to $\mathrm{MLS}_{\mathrm{B}}$ antibiotics ( $\mathrm{MLS}_{\mathrm{B}}$-resistant phenotype) and remains the most frequent mechanism of resistance. In general, genes encoding these methylases have been designated erm (erythromycin ribosome methylation). Expression of resistance to $\mathrm{MLS}_{\mathrm{B}}$ in staphylococci may be constitutive $\left(\mathrm{MLS}_{\mathrm{BC}}\right)$ or inducible $\left(\mathrm{MLS}_{\mathrm{Bi}}\right)$. When expression is constitutive, the strains are resistant to all MLS $_{\mathrm{B}}$ type antibiotics. When expression is inducible, the strains are resistant to 14- and 15-membered macrolides only [11,12].

Received on 24 August 2006; revised 8 December 2006.

Address for correspondence: Dr. Leandro Reus Rodrigues Perez. Fundação Faculdade Federal de Ciências Médicas de Porto Alegre. Rua Sarmento Leite 245/211. Zip code: 90050-170 Porto Alegre,RS, Brazil. Phone: 55-051-32248822. Fax: 55-051-32269756. E-mail address: leandro.reus@gmail.com..

The Brazilian Journal of Infectious Diseases

2007;11(2):186-188. (C) 2007 by The Brazilian Journal of Infectious Diseases and Contexto Publishing. All rights reserved.
For $\mathrm{MLS}_{\mathrm{Bi}}$ strains, erythromycin will induce production of the methylase, which allows clindamycin resistance to be expressed. Inducible clindamycin resistance can be detected with a simple disk approximation test, commonly referred as the $\mathrm{D}$ test [14]. For this test, an erythromycin disk is placed $15 \mathrm{mM}$ to $26 \mathrm{mM}$ (edge to edge) from a clindamycin disk in a standard disk diffusion test. Following incubation, a flattening of the zone in the area between the disks where both drugs have diffused indicates that the organism has inducible clindamycin resistance [14-16].

The purpose of this study was to characterize the antimicrobial susceptibility patterns (erythromycin, clindamycin and oxacillin) and to evaluate, according to the D test [17], all coagulase negative staphylococci from a collection of 200 clinical isolates from blood cultures that had the necessary characteristics for this study: resistance to erythromycin and susceptibility to clindamycin. We analyzed 200 consecutives clinical isolates of CoNS obtained from patients admitted in a general hospital, in Porto Alegre city, in Southern Brazil, between January and June 2002. All the isolates were obtained from blood cultures.

The samples were identified (only for species-level identification) through MicroScan, panel Pos-Combo 13 (Dade Behring - Deerfield, Illinois, USA). For selection criteria, a method for determining susceptibility to clindamycin (disk diffusion) was performed rather separated on the plate and so it was not confused with the performance of the D test itself.

The susceptibility tests - disks for the following agents at the amounts specified: $15 \mu$ g erythromycin, $2 \mu$ g clindamycin and $1 \mu$ g oxacillin (Difco Laboratories, Detroit, Mich.) - were performed by the agar disk diffusion method (Kirby-Bauer), according to the guidelines of the National Committee for Clinical Laboratory Standards.

D test Method - for this test, erythromycin disks were placed at $15 \mathrm{mM}$ and $26 \mathrm{mM}$ (edge to edge) from clindamycin disks, as recommended (NCCLS, 2004) on Muller-Hinton agar plate (Oxoid - Hampshire, England). Moreover, the disks also were placed at a distance of $10 \mathrm{~mm}$. According to evaluation criteria of NCCLS 2004, the flattened (positive test) or not 
(negative test) clindamycin zone between erythromycin and clindamycin disks was verified.

We performed the clindamycin induction test on CoNS that had the following profile: resistant or with intermediate resistance to erythromycin and susceptible to clindamycin, using routine antimicrobial susceptibility test. Twenty-seven CoNS of our collection $(\mathrm{n}=200)$ had this profile. S. aureus ATCC 25923 was used for quality control (QC) of clindamycin and erythromycin disks, according to the standard disk diffusion QC procedure. The susceptibility patterns for CoNS isolates are showed in the Table 1 . One hundred thirty-three (66.5\%) out of the isolates were oxacillin-resistant. The full and intermediate resistance were $63 \%$ and $3.5 \%$, respectively, for erithromycin and $53.5 \%$ and $1 \%$ respectively, for clindamycin. The phenotypic pattern compatible for realization of the D test was obtained by 27 CoNS isolates (13.5\% of the total). Thus, only 5 (18.5\%) were positive for inducible clindamycin resistance. The test was more visible when the erythromycin disk was placed $15 \mathrm{mM}$ or $10 \mathrm{mM}$ from the clindamycin disk. Positive D test reactions were showed when intermediate resistance to erythromycin was included as well as full resistance. In total, 5 positive reactions were observed, 3 (14.3\%) for full resistance and 2 (33.4\%) for intermediate resistance to erythromycin. We observed distinct species in these 5 positive D test CoNS isolates: 2 Staphylococcus epidermidis, 2 Staphylococcus haemolyticus and 1 Staphylococcus simulans. Both S. epidermidis (2) and S. haemolyticus (2) were carriers of the mecA gene - oxacillin-resistant, whereas $S$. simulans was not carrier (data not showed). The distances between disks more suitable to detection of the induction of resistance were $15 \mathrm{mM}$ (standard) and $10 \mathrm{mM}$ (no standard). The Figure 1 showed the induction of the resistance at a distance of $10 \mathrm{~mm}$.

Resistance in Gram-positive bacteria not only increases morbidity and mortality, but also the costs of management of hospitalized patients. Studies have indicated a great increase in the ratio of staphylococci resistance to MLS group and failure in the treatment with clindamycin in infections with microorganisms with inducible resistance to MLS group [17]. Reporting Staphylococcus spp. as susceptible to clindamycin without checking for inducible clindamycin resistance may result in inappropriate clindamycin therapy. As caution, to add comment of resistance based on detection of inducible clindamycin resistance has been proposed [18]. On the other hand, negative results for inducible clindamycin resistance confirm susceptibility to clindamycin and add comment that this Staphylococcus spp. does not demonstrate inducible clindamycin resistance in vitro [18].

The D test is acceptable for all Staphylococcus spp. including oxacillin-susceptible or oxacillin-resistant $S$. aureus or CoNS [18]. Many of the recently recognized methicillinresistant Staphylococcus aureus (MRSA) that cause community-associated infections have the msrA gene, and oral clindamycin may be a treatment option for these patients. In this case, these $S$. aureus strains are susceptible to clindamycin and do not present inducible resistance to this antimicrobial agent. Although clindamycin can be effective in some patients, it is not recommended to use it before conduct the $\mathrm{D}$ test [18].

An important fact in our study was that we incorporated clinical isolates of CoNS that presented a profile of intermediate resistance to clindamycin. In fact, 2 isolates that showed compatible profile with the realization of the $\mathrm{D}$ test (erythromycin-intermediate and clindamycin-susceptible), resulted in resistance to clindamycin and positive $\mathrm{D}$ test. This isolates were identified as Staphylococcus epidermidis and Staphylococcus haemolyticus. Outbreaks caused by multiresistent and offensive Staphylococcus epidermidis and Staphylococcus haemolyticus have been reported in various nosocomial settings, as well as in individual intensive care units (ICU) or other units within a hospital [19]. Save this results, our Staphylococcus spp. isolates have resistance levels lower than in other countries (data not published was showed in the $104^{\text {th }}$ General Meeting of the American Society for Microbiology, New Orleans, LA, 2004). Until now, there are not studies that report this test in Brazilian clinical isolates. This disk approximation test proved to be a good method to detect staphylococci strains with inducible clindamycin resistance. As demonstrated in the effectuated analyses, the method revealed to be adequate and viable for the evaluation of this phenotype of resistance when it was used $15 \mathrm{mM}$ (standard) and $10 \mathrm{mM}$ (no standard) of distance between the disks. At a distance of $26 \mathrm{mM}$ between the disks, macrolide resistance was not detected in 2 isolates $(1 \mathrm{~S}$. epidermidis and $1 S$. haemolyticus). In summary, the $\mathrm{D}$ test method revealed to be practical in the established conditions, being able to be used in the qualitative determination (phenotyping) of the resistance in coagulase negative staphylococci, mainly when the lowest standardized distance $(15 \mathrm{~mm})$ between disks was used. Additional advantages include decreased managements costs of treatment in resistant infections (by diagnostic confirmation), more rapidity in the results and its easy adaptation in the laboratorial routine.

The $\mathrm{D}$ test can be used as an auxiliary and alternative method to inducible clindamycin resistance detection in the routine of clinical laboratories. However, the confirmation of the erm gene in staphylococci strains with positive D test would assist in the standardization of the test (suitable distance between disks, sensitivity and specificity of the test). Moreover, the present study verified only 5 positive tests and a greater number would be required for validation of the interpretation of the distance between the disks.

\section{Acknowledgements}

The authors wish to thank Maria Beatriz and Rosângela for their support, bacteriology of the Irmandade Santa Casa de Misericórdia de Porto Alegre (ISCMPA), Fundação Faculdade Federal de Ciências Médicas de Porto Alegre (FFFCMPA), Conselho Nacional de Desenvolvimento Científico e Tecnológico (CNPq) and Coordenação de Aperfeiçoamento de Pessoal de Nível Superior (CAPES), Brasília, Brazil. 
Table 1. Antimicrobial susceptibility patterns among coagulase negative staphylococci isolates

\begin{tabular}{lccc}
\hline Pattern & \multicolumn{2}{c}{ Antimicrobials } \\
\cline { 2 - 4 } & Oxacillin (\%) & Erythromycin (\%) & Clindamycin (\%) \\
\hline Resistant & $133(66.5)$ & $126(63)$ & $107(53.5)$ \\
Intermediate & - & $07(3.5)$ & $02(1)$ \\
Susceptible & $67(33.5)$ & $67(33.5)$ & $91(45.5)$ \\
Total & $200(100)$ & $200(100)$ & $200(100)$ \\
\hline
\end{tabular}

Figure 1. D test positive result for $S$. epidermidis strain (distance of $10 \mathrm{mM}$ between disks).

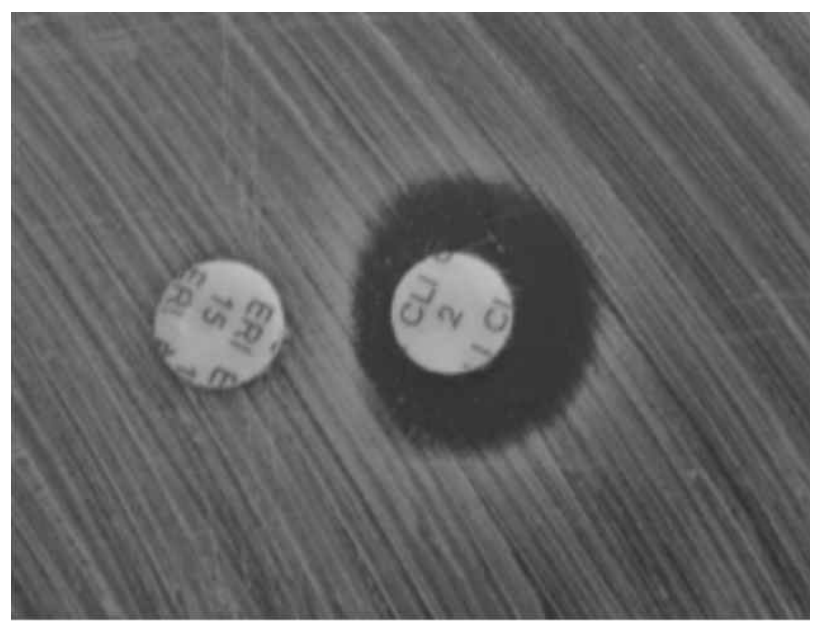

\section{References}

1. Fluit A.C., F.-J. Schmitz, M. E. Jones, et al. Verhoef for the SENTRY Participants Group. (1999). Antimicrobial resistance among community-acquired pneumoniae isolates in Europe: First results from the SENTRY antimicrobial surveillance program. Int J Infect Dis 1997;3:153-6.

2. Fluit A.C., M.E. Jones, F.-J. Schmitz, et al. Bacteremia in European hospitals, incidence and antimicrobial susceptibility. Clin Infect Dis 2000;30:454-60.

3. Fluit A.C., M.E. Jones, F.J. Schmitz, et al. Antimicrobial resistance among Urinary Tract Infection (UTI) isolates in Europe: Results from the SENTRY Antimicrobial Surveillance Program 1997. Antonie Leeuwenhoek 2000;77:147-52.

4. Jenssen W.D., S. Thakker-Varia, D.T. Dubin, M.P. Weinstein. Prevalence of macrolides-lincosamides-streptogramin B resistance and erm gene classes among clinical strains of staphylococci and streptococci. Antimicrob Agents Chemother 1987;31:883-8.

5. Arthur M., C. Molinas, C. Mabilat, P. Courvalin. Detection of erythromycin resistance by the polymerase chain reaction using primers in conserved regions of erm rRNA methylase genes. Antimicrob Agents Chemother 1990;34:2024-6.

6. Eady E.A., J.I. Ross, J.L. Tipper, et al. Distribution of genes encoding erythromycin ribosomal methylases and an erythromycin efflux pump in epidemiologically distinct groups of staphylococci. J Antimicrob Chemother 1993;31:211-7.
7. Allignet J., N. El Solh. Diversity among the Gram-positive acetyltransferases inactivating streptogramin A and structurally related compounds and characterization of a new staphylococcal determinant, vatB. Antimicrob Agents Chemother 1995;39:2027-36.

8. Allignet J., S. Aubert, A. Morvan, N.El Sohl. Distribution of genes encoding resistance to streptogramin A and related compounds among staphylococci resistant to these antibiotics. Antimicrob Agents Chemother 1996;40:2523-8.

9. Allignet J., N. Liassine, N. El Sohl. Characterization of a staphylococcal plasmid related to pUB110 and carrying two novel genes, vatC and $v g b B$, encoding resistance to streptogramins A and B and similar antibiotics. Antimicrob Agents Chemother 1998;42:1794-8.

10. Jensen L.B., N. Frimondt-Moller, F.M. Aarestrup. Presence of erm gene classes in Gram-positive bacteria of animal and human origin in Denmark. FEMS Microbiol Lett 1999;170:151-8.

11. Leclercq R., R.B. Giannattasio, H.J. Jin, B. Weisblum. Bacterial resistance to macrolide, lincosamide and streptogramin antibiotics by target modification. Antimicrob Agents Chemother 1991;35:1267-72.

12. Weisblum B. Resistance to macrolide-lincosamide-streptogramin antibiotics, p. 682-98. In: V.A. Fischetti (ed.), Gram-positive pathogens. American Society for Microbiology, Washington, D.C., 1999.

13. Eady E.A., J.I. Ross, J.L. Tipper, et al. Distribution of genes encoding erythromycin ribosomal methylases and an erythromycin efflux pump in epidemiologically distinct groups of staphylococci. J Antimicrob Chemother 1993;31:211-7.

14. Fiebelkorn K.R., Crawford S.A., McElmeel M.L., Jorgensen J.H. Practical disk diffusion method for detection of inducible clindamycin resistance in Staphylococcus aureus and coagulasenegative staphylococci. J Clin Microbiol 2003;41:4740-4.

15. Sanchez, M. L., K. K. Flint, and R. N. Jones. Occurrence of macrolidelincosamide-streptogramin resistances among staphylococcal clinical isolates at a university medical center. Is false susceptibility to new macrolides and clindamycin a contemporary clinical and in vitro testing problem? Diagn Microbiol Infect Dis 1993;16:205-13.

16. Fiebelkorn K.R., Crawford S.A., McElmeel M.L., Jorgensen J.H. Practical disk diffusion method for detection of inducible clindamycin resistance in Staphylococcus aureus and coagulasenegative staphylococci. J Clin Microbiol 2003;41:4740-4.

17. Panagea S., Perry J.D., Gould F.K. Should clindamycin be used as treatment of patients with infections caused by erythromycinresistant staphylococci? J Antimicrob Chemother 1999;44:581-2

18. National Committee for Clinical Laboratory Standards - NCCLS. Performance Standards for Antimicrobial Disk Susceptibility Tests. M100-S14. Wayne, PA: National Committee for Clinical Laboratory Standards, 2004.

19. National Nosocomial Infections Surveillance (NNIS) System. (1999). National Nosocomial Infections Surveillance (NNIS) System Report, data summary from January 1990-May 1999, issued June 1999. Am J Infect Control 1999;27:520-32. 